\title{
FUNDAMENTACIÓN TEÓRICA Y METODOLÓGICA DEL COMPORTAMIENTO DEL CONSUMIDOR EN CAFETERÍAS - PERSPECTIVA DE LA RACIONALIDAD
}

\section{METHODOLOGICAL AND THEORIC FOUNDATION OF THE CONSUMER'S BEHAVIOUR IN CAFETERIAS}

\begin{abstract}
Viviana Alexandra Sacoto Castillo, Mgs. Magíster en Economía con mención en Finanzas y Proyectos Corporativos (Ecuador). Docente Titular de la Facultad de Ciencias Económicas de la Universidad de Guayaquil,

Ecuador. viviana.sacotoc@ug.edu.ec
\end{abstract}

Juan Antonio Jimber Del Río, PhD. Doctor en Ciencias Sociales y Jurídicas de la Universidad de Córdoba (España). Docente Ayudante Doctor de la Facultad de Derecho y Ciencias Económicas y Empresariales de la Universidad de Córdoba, España. jjimber@uco.es

Leonor Pérez Naranjo, PhD.

Doctora en Ciencias Económicas y Empresariales de la Universidad de Córdoba (España).

Profesora Contratada, Doctora Interina en el Área de Organización de Empresas, Coordinadora del Itinerario Conjunto del Grado en Turismo y Traducción e Interpretación de la Universidad de Córdoba, España. Iperez@uco.es

\section{ARTÍCULO DE INVESTIGACIÓN}

Recibido: 3 de septiembre de 2018.

Aceptado: 21 de noviembre de 2018.

\section{RESUMEN}

El artículo presenta la fundamentación teórica y metodológica preliminar de los factores influyen en el comportamiento de compra en cafeterías como una evaluación desde la perspectiva de la racionalidad. Se presentan los sustentos teóricos e investigaciones previas con la metodología propuesta y variables. Los estudios se han basado en recolección de datos 
mediante encuestas aplicadas en cafeterías, hoteles tradicionales y temáticos que evalúan principalmente las siguientes variables: percepciones, lealtad, personalidad, ambiente del negocio, entre otros. La metodología que se propone en la investigación es cuantitativa, correlacional y transversal, con recolección de datos a través de la aplicación de encuestas, por medio de un piloto y se demostrará la validez y confiabilidad del instrumento (medida de Alfa de Cronbach). La encuesta se aplicará a un conjunto de consumidores a través de un muestreo aleatorio y sistemático. Por medio de análisis factorial exploratorio se medirán los datos, para finalizar con la comprobación del modelo por medio de ecuaciones estructurales en el paquete informático SPSS.

Palabras clave: Percepciones, consumidor, satisfacción, lealtad, racionalidad

\section{ABSTRACT}

The article presents the preliminary theoretical and methodological foundation of the factors influencing shopping behavior in cafeterias as an evaluation from a rationality perspective. The theoretical and previous investigations with the proposed methodology and variables are presented. The studies have been based on data collection through surveys applied in cafeterias, traditional and thematic hotels that mainly evaluate the following variables: perceptions, loyalty, personality, business environment, among others. The methodology proposed in the research is quantitative, correlational and transversal, with data collection through the application of surveys, by means of a pilot and the validity and reliability of the instrument will be demonstrated (Cronbach's Alpha measurement). The survey will be applied to a set of consumers through random and systematic sampling. By means of exploratory factor analysis, the data will be measured, to end with the verification of the model by means of structural equations in the SPSS software package.

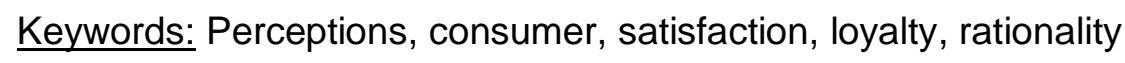

\section{INTRODUCCIÓN}

La Ciencia Económica tiene como objetivo fundamental el estudio del problema económico en la sociedad, es decir la relación existente entre las necesidades y la distribución eficiente de los recursos que se consideran escasos, para lo cual se analizan los agregados macroeconómicos, pudiendo así determinar la situación global de la economía; mientras que 
desde una perspectiva microeconómica se analiza el comportamiento e interacción de consumidores y productores. Dentro del análisis del comportamiento del consumidor se observan determinados factores que influyen en el momento de la compra de bienes y servicios en los mercados, para lo cual se utilizan supuestos que permiten modelar y tratar de comprender las decisiones de compra.

Los factores que influyen en la demanda generalmente utilizados son: precio del bien analizado, ingresos, precio de los bienes sustitutos, gustos y preferencias, número de consumidores; y los principales supuestos aplicables dentro de Microeconomía es la racionalidad del consumidor y en función de aquello el individuo busca maximizar sus niveles de satisfacción por medio del consumo de bienes y servicios que conforma su cesta o canasta de mercado; entendiéndose por aquella que agrupa un conjunto de bienes y servicios necesarios para satisfacer sus necesidades en un período determinado.

En tal contexto, a partir del supuesto de racionalidad se asume que el consumidor realiza sus compras de manera racional y basándose en criterios de maximización; sin embargo, al abordar concepto de incentivos, impulsividad, satisfacción, percepción, tipos de personalidad, entre otros; se puede analizar comportamientos que no siempre responden a los supuestos generalmente estudiados.

El hecho relevante de análisis propuesto se basa en cuanto a la tendencia de consumo que presentan las personas y su evolución, ya sea en sus hábitos, gustos o preferencias. El entendimiento de las causas que responden a dichos cambios en las tendencias de consumo podrían explicar patrones de comportamiento que pueden ser utilizados para mejorar las estrategias de cafeterías e incluso para adoptarlo como punto de partida para el análisis de otros negocios.

Para el desarrollo de la investigación se analizarán diversas teorías y sustentos de escuelas económicas, que han venido presentando planteamientos a lo largo de la historia, y que han evidenciado avances en la ciencia. Entre las principales escuelas económicas se encuentran: Mercantilista, Fisiócrata, Clásica, Neoclásica, Keynesiana, Marxista y Monetarista. Posteriormente, surge la Escuela Marginalista (1870) con Jevons, Menger y Walras, cuyo pilar de análisis se encontraba focalizado la teoría subjetiva del valor y adquiere así mayor relevancia el consumo, la utilidad; y se reconoce la utilidad marginal decreciente; para finalmente fijar el interés de estudio en la conducta de los individuos. 
Se han presentado aportes o contribuciones al estudio iniciado por la Escuela Marginalista cuyo grado de ampliación o profundidad en el estudio ha continuado con atención al comportamiento del individuo y es así que el sicólogo Daniel Kahneman (2002) Premio Nobel de Economía, consideró el sustento de análisis en el estudio del ser humano como un acto dentro de la economía, pero quien de forma individual adopta posiciones frente a sus decisiones de tipo económica. Él observó comportamientos y fueron valorados surgiendo así situaciones no consideradas en detalle por la ciencia económica hasta ese momento. Otro referente actual en cuanto al estudio en finanzas conductuales es Richard Thaler a quien se le otorgó el Nobel de Economía en el año 2017, en cuyas investigaciones ha realizado aportes a la economía conductual. Al respecto contribuye Jeannot (2006):

A Kahneman y Tversky les otorgaron el Nóbel "por haber introducido en economía avances de la investigación psicológica, especialmente en lo que respecta al juicio humano y la toma de decisiones bajo incertidumbre" (Real Academia Sueca de Ciencias, 2002). En efecto, sus experimentos permitieron refrendar la paradoja de Allais, en el sentido de cuestionar a la teoría de la utilidad esperada que era -y esusada mayoritariamente por la corporación de los economistas neoclásicos. Esta teoría de la utilidad postula que los individuos son capaces de ordenar perfectamente todos los estímulos que enfrentan y elegir entre ellos para maximizar su satisfacción (p. 57).

Existen estudios de mercado que permiten a los empresarios disponer de información que le permitirá conocer las necesidades, su predisposición al consumo, precios que estarían dispuestos a pagar por el bien o servicio, entre otros, sin embargo no se consideran factores adicionales que pueden influir en las compras y que en ciertas ocasiones son valorados en la ejecución del negocio, desde las encuestas de servicio al cliente para medir niveles de satisfacción; pudiendo haber determinado con anterioridad a qué otros factores pudieran estar respondiendo el consumo, adoptando estrategias desde el inicio, en función de las respuestas. La relevancia de la investigación se basa en la oportunidad de aplicación de estrategias oportunas de los empresarios ante el conocimiento de comportamientos y motivaciones de actuación de los consumidores en los mercados.

En diversas investigaciones se han presentado estudios de comportamiento del consumidor enfocados en aquellas variables que generan lealtad en los clientes, niveles de satisfacción, calidad, percepción de precios, decoración, ambiente, condiciones ambientales. Las 
investigaciones fueron aplicadas en diferentes contextos, relacionadas a turismo, consumo de restaurantes tradicionales y de lujo, en menor medida en cafeterías; sin embargo, no se evidencian muchos estudios aplicados al análisis de variables sicológicas en la valoración del comportamiento del consumidor específicamente en cafeterías.

La percepción, psicología de la conducta, psicología económica, formalización de modelos, incentivos, forman parte de los estudios realizados, así como también en el levantamiento inicial de fundamentación teórica, en el artículo se abordan estudios previos se han basado en recolección de datos mediante encuestas aplicadas en cafeterías, hoteles tradicionales y temáticos que evalúan principalmente las siguientes variables y constructos: percepciones, lealtad, personalidad, ambiente del negocio, experiencia sensorial entre los principales.

El artículo aborda la fundamentación teórica y metodológica preliminar de los factores influyen en el comportamiento de compra en cafeterías, como una evaluación desde la perspectiva de la racionalidad.

\section{REVISIÓN TEÓRICA}

\subsection{Aproximación teórica al comportamiento del consumidor.}

El comportamiento del consumidor ha sido ampliamente estudiado desde diversas perspectivas en donde se han abordado definiciones relacionadas tales como: satisfacción, percepción, motivaciones, incentivos, emociones, lealtad; entre otros términos que describen comportamientos. Ciertos autores coinciden en que existen elementos de psicología que son factores importantes que permiten entender de mejor forma el comportamiento del consumidor, es así que, Endo \& otros (2017), afirman:

El estudio del campo de la psicología tiene elementos importantes que pueden ayudar a comprender mejor el comportamiento del consumidor. Conceptos tales como los niveles de atención selectiva, percepción y memoria, ampliamente utilizados en Neuropsicología, pueden ser muy efectivos cuando se traen al campo de la comunicación, en la medida en que subyace y da consistencia a los temas que los profesionales en esta área a menudo defienden en Una manera meramente subjetiva. (p.3) 
Se abordarán definiciones que parten de la sicología, por tratarse de comportamientos de individuos que involucran los procesos cognitivos y con ello se podrá predecir de manera más cercana a la realidad aquellas motivaciones y decisiones que conllevan el consumo.

Al iniciar el análisis del comportamiento del consumidor, se debe conocer diferentes definiciones de la consecuencia básica del consumo, es decir de la satisfacción que se genera, al respecto se expresa: "La satisfacción del consumidor se puede definir como un juicio, bien de naturaleza cognitiva o bien de carácter afectivo o emocional, que deriva de la experiencia del individuo con el producto o servicio" (Oliver, 1997). (Devesa y otros 2010, p.4), la satisfacción del consumidor la define como un bien cognitivo en donde existe una conjugación de emociones, permitiendo que las personas obtengan experiencias del producto, entendiéndose que pueden tener manifestaciones positivas o negativas del consumo.

La satisfacción desde la perspectiva de (Moliner, Berenguer y Gil, 2001) la define:

Un estado psicológico que resulta cuando se le asocia una emoción, provocando una reinterpretación del sentimiento relacionado con la experiencia de consumo. Esta conceptualización no supone una alternativa frente a la perspectiva cognitiva, sino un complemento que enfatiza otros centros de interés. Por lo tanto, no se niega que la satisfacción sea una consecuencia de un proceso cognitivo (de naturaleza variada), sino que éste, además se asociará con distintas emociones (p.2).

De acuerdo a los autores se manifiesta la satisfacción mediante un sentimiento como consecuencia del consumo; en donde se logra complementar con los "centros de interés", en donde se genera además de un proceso cognitivo, la asociación de emociones variadas. Esta definición mantiene como base de la definición anterior, la presencia de procesos cognitivos y le adiciona los resultados de emoción que manifiesta el individuo por su experimentación en el consumo.

(Forgas, Moliner, Sanchez, y Palau, 2011) al analizar la satisfacción del consumidor, afirman: La satisfacción es el sentimiento del consumidor de que el consumo provee un resultado contra un estándar de placer versus no placer. Para que la satisfacción afecte a la lealtad una satisfacción frecuente o acumulada es necesaria, de tal manera que los episodios de satisfacción individual se agreguen y mezclen (p.2).

Los autores expresan a la satisfacción como parte de resultados obtenidos en base a un estándar de "placer" y "no placer" que identifica cada individuo entorno al consumo de un bien; 
es decir que la generación de satisfacción responde a estándares y agregan que también forma parte de una acumulación del placer para mantener la lealtad. Se introduce adicionalmente la relación entre la satisfacción y la lealtad que se genera en el consumo de un bien o servicio, siempre que se cumpla con el estándar previamente establecido. Entorno a la diversidad de conceptos, se presenta a la satisfacción desde las definiciones que afirman que generalmente se presentan tres elementos comunes, "la satisfacción es una respuesta post-compra del consumidor; ésta está referida a unos focos determinados; y se da en un momento concreto" (Ruiz y Palaci, 2011, p.1); es decir que se enfoca a la satisfacción de acuerdo al momento en que se experimenta la compra y su respuesta posterior, con análisis de determinados factores.

Con base en las definiciones de satisfacción que se generan del consumo, existen términos relacionados que explican la respuesta que tiene el consumidor ante su experimentación, entre ellas se encuentra la lealtad, siendo así que se define la lealtad como: "El compromiso de más alto nivel que supone la transición de una predisposición favorable (lealtad afectiva) a un compromiso de compra repetida (lealtad conativa) como paso previo a la acción de compra Oliver (1999)" (Forgas y otros, 2011, p.2), es decir que la lealtad genera repetición en el consumo de un bien o servicio'; siendo importante mantener los niveles de satisfacción esperados o estándar de los consumidores para garantizar el compromiso de consumos futuros. Para cumplir con las expectativas y estándares de los consumidores es necesario identificar cuáles son los factores que influyen en el consumo de un determinado bien o servicio y los incentivos que generan lealtad.

Desde la definición de los tipos de lealtad, se distinguen dos tipos, "la lealtad verdadera y la lealtad espuria, siendo la primera el proceso que predispone favorablemente a un consumidor a efectuar un acto reiterado de compra, y siendo la segunda fruto de la casualidad y/o de la preferencia por conveniencia Oliver (1999)" (Forgas y otros, 2011, p.2) entendiéndose los tipos de lealtad, entonces las empresas deberían perseguir la lealtad verdadera que permita que el consumidor realice compras reiteradas, y no únicamente que sean consecuencia de la casualidad.

De acuerdo a la definición de niveles de lealtad, expresado por (Forgas y otros, 2011, p. 2) se presenta a continuación una tabla que resume los niveles de lealtad: 
Tabla 1. Niveles de Lealtad.

\begin{tabular}{|c|c|}
\hline Niveles de Lealtad & Definición \\
\hline \multirow[t]{3}{*}{ 1.Lealtad Cognitiva } & $\square$ Vínculo más débil de la fidelidad \\
\hline & $\begin{array}{l}\square \text { Lealtad basada en elementos } \\
\text { funcionales: precio, calidad, } \\
\text { programa de puntos. }\end{array}$ \\
\hline & $\begin{array}{l}\square \text { Puede ser superado por } \\
\text { competidores. }\end{array}$ \\
\hline 2.Lealtad Afectiva & $\begin{array}{l}\square \text { Basada en los sentimientos y } \\
\text { emociones generados por la } \\
\text { compañía o marca en el cliente }\end{array}$ \\
\hline 3.Lealtad conativa & $\begin{array}{l}\square \text { Intención de comportamiento } \\
\square \text { Relacionada con la recompra o con la } \\
\text { recomendación }\end{array}$ \\
\hline 4.Lealtad comportamental & $\begin{array}{l}\square \text { Un comportamiento concreto y } \\
\text { que es consecuencia de los tres } \\
\text { niveles anteriores. }\end{array}$ \\
\hline
\end{tabular}

Fuente: Elaboración propia a partir de Forgas, Moliner, Sanchez y Palau (2011).

De acuerdo a lo expresado por los autores citados en la tabla anterior, se presentan 4 niveles de lealtad, los cuales nacen como una consecuencia de la experiencia positiva en el consumo de un determinado bien o servicio; siendo así, que la lealtad cognitiva la consideran como un vínculo débil de fidelidad, ya que puede ser superado por otros vendedores dentro del mercado. La lealtad afectiva, se genera en base a sentimientos que responden positivamente a la marca, en tanto la lealtad conativa, está relacionada con una reacción del comportamiento para comprar en el futuro e incluso tener la predisposición de recomendar el bien o servicio, y finalmente la denominada lealtad comportamental que es una agrupación de los tres niveles mencionados anteriormente.

Las emociones en su nivel más básico, desde el campo estrictamente sicológico, tiene argumentaciones y define ciertos comportamientos del individuo, siendo así que Borrás y otros (2004) afirman: 
En tanto que asumimos las diferentes emociones que experimentamos como parte natural de nuestra rutina diaria, difícilmente las cuestionamos, problematizamos o pensamos siquiera en ellas. Gestionarlas es la vida misma de la que cada cual se ocupa, ¿acaso hay un sistema de control más eficaz? Nos sentimos impelidos a perfeccionarlas, conocerlas (con psiquiatras y manuales de autoayuda varios) e invertir lo que sea para poder vivirlas y experimentarlas en plenitud, para lograr una vida que valga la pena de ser vivida. Nos inquieta sentir algo que no podemos catalogar fácilmente como una emoción conocida, porque ello puede ser peligroso, o síntoma de que no estamos siendo auténticos, o de que nos estamos engañando a nosotros mismos, o de que no estamos en consonancia con nuestra naturaleza (p. 182).

Las emociones forman parte de la definición de satisfacción desde el enfoque de análisis de varios autores, por lo cual es importante definirla, en base a lo expresado por (Matos y Martín, 2012) las emociones se definen:

Respuestas provocadas por un estímulo específico, generalmente tienen la característica de ser intensas y al tener una mayor duración queda como recuerdo de la emoción en el pasado, es decir, estas son almacenadas en la memoria y recobradas posteriormente durante la exposición de la experiencia. (p.7)

De acuerdo a la definición, las emociones son repuestas del individuo con respecto a estímulos, teniendo dos características importantes para permanecer en el tiempo: intensidad y duración; con la finalidad de almacenarse en la memoria y activarse en cuanto exista la posibilidad de volver a experimentarlas. Esta definición conduce a comprender al individuo para que las empresas mediante diversos estímulos puedan generar emociones positivas (intensas y de extensa duración) que se traducen en satisfacción y con ello generar lealtad que al llegar a desarrollarse el máximo nivel (lealtad comportamental) poder generar compromisos con la marca y por ende con la compra de los productos o servicios consumidos.

Dentro de las dimensiones en cuanto al agrado y activación de emociones, según lo expresado por (Matos y Martín, 2012) las conceptualizan de acuerdo a lo presentado:

La dimensión agrado es un sentimiento subjetivo de lo agradable o desagradable, haciendo referencia al nivel en el cual una persona se siente bien o mal (e.g., felizinfeliz) en una determinada situación; y la dimensión activación como un estado subjetivo de sentimiento activado o desactivado (e.g., animado-decaído), refiriéndose a la extensión en la cual una persona se siente estimulada y activa. (p.8) 
Dentro de las emociones se expresa el agrado, en donde se manifiesta que es un estado subjetivo del individuo, es decir que para algunos puede algún estimulo resultar agradable, mientras que para otra persona ser desagradable, lo cual relativiza la predicción del comportamiento, sin embargo introduce como parte de la valoración la evaluación del entorno, siendo así que ( Matos y Martín, 2012) manifiestan: "se evalúa el entorno por medio de indicadores intangibles, por ejemplo, los indicadores de la calidad ambiental percibida por los consumidores"(p.7).

La motivación y percepción son parte del estudio del comportamiento del consumidor, por lo cual es importante definirla, al respecto la definen como: "la necesidad o el deseo que dirige y energiza la conducta hacia una meta (Arana y otros, 2010) (Sergueyevna Golovina \& Mosher Valle, 2013, p.2) y que junto a las emociones forma parte cognitiva de la mente humana. Mientras que la percepción de acuerdo a lo expresado por Oviedo et. al (2004):

La percepción como un proceso de extracción y selección de información relevante encargado de generar un estado de claridad y lucidez consciente que permita el desempeño dentro del mayor grado de racionalidad y coherencia posibles con el futuro circundante. (p. 89)

La motivación de un individuo se enfoca hacia una necesidad o deseo para alcanzar una meta, que al relacionarlo con el comportamiento del consumidor se acerca a la reacción del individuo ante estímulos, los cuales van a estar acordes a sus expectativas y es allí donde se debe analizar la percepción para tratar de comprender el proceso interno que realiza para la compra de bienes o servicios.

Con respecto a la conducta de compra, Berenguer (2006) expresa:

Asumimos como hipótesis de partida que la conducta de compra y de consumo está influida por un proceso de naturaleza interna -y por lo tanto no observable- al que se suele denominar motivación. De ahí que pueda afirmarse que la motivación es lo que se denomina un concepto hipotético, es decir, un concepto que inferimos porque se aprecia un cambio de estado en la conducta observable de un individuo. Al ocurrir en el interior de un sujeto no podemos verlo, pero se puede inferir a partir de su conducta. (p. 247)

Las conductas de compra tienen influencia interna en el individuo, por lo cual Berenguer (2006) lo denomina motivación y en tal sentido no se puede tener control exacto ni certeza; sin 
embargo, puede inferirse conductas adoptadas frente a determinados hechos, e incluso económicas, así mismo el autor expresa la teoría motivacional que incentivan al individuo a actuar y así como otros autores introduce aspectos ambientales en la toma de decisiones del individuo, así como Berenguer (2006) "Este enfoque, denominado conductista, considera al individuo como una "caja negra" que reacciona de forma automática a los estímulos ambientales" (p. 247).

\subsection{Investigaciones previas del Comportamiento del Consumidor.}

La teoría Microeconómica presenta el análisis de la utilidad que obtiene el individuo en términos de niveles de satisfacción al consumir un determinado bien y basado en supuestos, se ha considerado que el consumidor es racional y realiza sus elecciones de compra analizando la maximización de beneficios, de acuerdo a lo expresado por Marshall, el consumidor adopta decisiones de compra de acuerdo a cálculos racionales, económicos y conscientes. Otros aportes teóricos de diversas áreas, entre ellas la psicoanalítica, en donde Freud, manifiesta que el ser humano tiene necesidades instintivas que podrían resultar en ciertos casos inaceptables socialmente; sin embargo, las limitaciones del estudio radican en determinar cuáles son los incentivos que presentan los individuos de necesidades insatisfechas que las pueden representar a través de las compras que realizan.

De acuerdo a ciertos estudios se ha evidenciado que el comportamiento del consumidor, responde al estudio de una economía positiva y en otros casos abordando diferentes enfoques, siendo así que aporta Jeannot, (2006):

Un comportamiento real es aquel que se verifica empíricamente: en cambio, un comportamiento racionalizado es aquel bosquejado por un investigador creyente en que el conocimiento de la realidad se basa en la razón, eventualmente estadística, pero no en los hechos. La utilización del "como si" (as if) es una aplicación del racionalismo metodológico que sustenta el individualismo del mismo carácter. Si bien este ismo no puede aplicarse a los clásicos de la economía, fueron los neoclásicos quienes con posterioridad, adscribieron la economía al racionalismo metodológico, tanto en la versión de la economía positiva (Friedman), como en la teoría pura (expectativas racionales y utilidad esperada). (p.57) 
Algunos autores consideran al consumo como una emoción, siendo así que Borrás y otros (2004) afirman:

Por fin se puede uno emocionar consumiendo emociones. El consumo es la emoción extrema, la emoción de emociones. La sociedad fundamentada económicamente en el consumo no requiere tan sólo de inventores apasionados por su trabajo y ciegos la mayor parte del tiempo a las consecuencias de éste -como la bomba atómica o la invención del telar a vapor-; además necesita estructuras de deseo imbuidas en sus unidades de consumo. (p. 192)

El autor expresa que los consumos generan emociones, así como también realiza una crítica a la racionalidad del consumidor, puesto que no siempre responde al comportamiento del "homo economicus", sino que la relaciona a la presencia de emociones en el ser humano.

La emoción del consumo sirve adecuadamente a su fin de mantener el engranaje de la economía en funcionamiento. Cuando los economistas utilizan su modelo preferido, el homo economicus hinchado de racionalidad, no se dan cuenta que éste ya acabó, que pudo ser útil cuando se trataba de explicar conductas de supervivencia en un entorno hostil como la industrialización, pero que no puede explicar cómo se comporta una persona educada en el consumo. Para el consumo se necesita la emoción. Una nueva especie, el homo emotionalis, se adecua más a la nueva situación. (p. 192)

También existen contribuciones, en cuanto al estudio del consumidor responsable, en donde se presentan críticas y se incorporan otros factores que influyen en la compra de bienes o servicios, entre ellos se encuentra la emoción y la influencia de grupos de referencia, a lo que algunos autores denominan el análisis de la psicología social, siendo así que Borrás y otros (2004) afirman:

Desde la psicología social hemos de responder a esta pregunta con una cierta prudencia. Por supuesto, existe una influencia obvia de las empresas, grupos de poder e instituciones, pero esta influencia también es ejercida por otros grupos, como son los vecinos, los amigos y la familia, y no conocemos el peso de cada grupo de influencia. Probablemente los grupos primarios sean más influyentes que los medios de comunicación, pero al mismo tiempo tenemos que considerar que las personas que componen nuestros grupos primarios también están al alcance de la influencia de los medios. Por otro lado, toda decisión que se toma tiene lugar en un contexto 
discursivo en el que los medios de comunicación desempeñan un papel importante, porque aportan constantemente argumentos y elementos de problematización. Hay que tener en cuenta que la libertad no puede considerarse en abstracto como una característica esencial y no problemática del ser humano. Toda decisión se toma siempre después de una consulta, implícita o explícita, más o menos consciente, a las comunidades de referencia del individuo. (p. 246)

El consumo se manifiesta como una necesidad de satisfacer necesidades, en donde también se consideran a los deseos como parte del estudio, porque están delimitados de acuerdo a prioridades del individuo, siendo importante identificar el objeto de cada elección de consumo, así como Huerga (2012) expresa:

El primer aspecto que hay que recordar es que el individuo presenta una serie de necesidades y deseos diferenciados entre sí, que persigue satisfacer de forma simultánea o progresiva, y que están organizados en alguna clase de estructura compleja, con distintos grados de prioridades. Además, lo normal es que cada bien -entendido de forma genérica, como cualquier "objeto" potencial de elecciónpresente múltiples características y atributos (Lancaster, 1966), que pueden afectar -de forma positiva o negativa, y en distinta magnitud- a diferentes necesidades y deseos del ser humano. (p.51)

En las investigaciones que se han efectuado sobre comportamiento de consumidores en diversos negocios, se ha evidenciado la base conceptual que los sustentan, la determinación de variables de estudios, metodología utilizada y los resultados de las investigaciones.

Lee (2018) en la investigación que realizó sobre los factores que influyen en la experiencia de consumo en restaurantes de un mall, entre ellos se encuentran: la imagen de la marca, la satisfacción y la lealtad del cliente, en donde evidenció que las variables definidas se encontraban relacionadas, para ellos corrieron 360 encuestas entre clientes que visitaron un restaurante y aplicaron análisis factorial confirmatorio y modelado de ecuaciones estructurales (SEM). Entre los principales hallazgos de la investigación Lee (2018) lo siguiente:

Estos esfuerzos contribuirán a desarrollar una imagen de marca positiva e influirán directamente en la lealtad de los clientes. Entre las relaciones con las pistas de mercadotecnia experiencial y la satisfacción, las emociones y los reconocimientos 
son impactos significativos en la satisfacción. Además, la emoción fue la mayor influencia en la pista de marketing experiencial. (p. 117) Traducción propia.

Heesup \& Kisang (2009), en la investigación que realizaron en restaurantes para determinar los factores de influencia que permiten generar satisfacción y lealtad al cliente, determinaron que entre ellos se encuentran: el entorno físico, percepción de precios, satisfacción del cliente y lealtad, en donde se utilizaron 279 encuestas que evidenciaron que la decoración (una de las variables de entorno fisico) influyó en la percepción de precios. El modelo utilizado fue por medio de ecuaciones estructurales. Heesup \& Kisang (2009) expresan:

Este estudio se centra únicamente en las relaciones entre el entorno físico, el precio, la satisfacción del cliente y la lealtad. El modelo propuesto se puede ampliar para incluir la teoría de interacción social y diversas respuestas internas de los clientes. La investigación indica que todas las interacciones sociales, como las interacciones de los clientes y los empleados, y la formación de relaciones en los servicios interpersonales se ven muy afectadas por las condiciones ambientales. (p. 506) Traducción propia.

El estudio de Chen \& Peng (2018), analiza las intenciones de consumidores para comer en restaurantes de lujo, en donde valoran una muestra de 361 encuestados para observar los comportamientos de las variables: valor funcional percibido, valor percibido simbólico / expresivo y valor hedónico.

En la revisión teórica de diversos autores se sustentan las variables relacionadas a satisfacción y lealtad de los clientes, que permite establecer estudios futuros y la investigación de variables que influyen y se relacionan en el modelo a plantearse. En el mismo orden que se presenta la revisión de investigaciones previas, se consolida en la Tabla 2 las variables analizadas en dichos estudios:

Tabla 2. Variables analizadas en estudios Previos relacionados.

\begin{tabular}{lll} 
Autor (es) & Tipo de negocio & $\begin{array}{c}\text { Principales Variables de } \\
\text { estudio }\end{array}$ \\
\hline Lee $(2018)$ & Restaurante & - Imagen de la marca \\
& & Satisfacción \\
& & Lealtad del cliente \\
\hline
\end{tabular}




\begin{tabular}{lll}
\hline Heesup \& Kisang & Restaurante & Entorno físico \\
(2009), & & Percepción de \\
& Preciós \\
& & Satisfacción \\
& & Lealtad \\
\hline Chen \& Peng & Restaurante & Valor funcional \\
(2018) & & percibido \\
& $\bullet$ & Valor percibido \\
& simbólico / expresivo \\
\hline & $\square$ & Valor hedónico \\
\hline Nama \& Timothy Restaurante & Calidad \\
Jeonglyeol (2011) & servicio \\
& $\square$ & Expectativa \\
& $\square$ & Precio \\
&
\end{tabular}

Fuente: Elaboración propia.

\section{MATERIALES Y MÉTODOS}

La investigación planteada tendrá un enfoque cuantitativo y transversal, cuyo alcance será correlacional, ya que analizará la relación entre variables, las mismas que surgen de la fundamentación teórica e investigaciones previas y basa su desarrollo en la organización inicial de información de manera lógica. Con una revisión futura se definirán las variables a relacionarse, de aquellas que se presentan en investigaciones previas, entre ellas se encuentran: niveles de satisfacción, calidad, percepción de precios, ambiente, personalidad, condiciones ambientales. Las investigaciones previas fueron aplicadas en diferentes contextos, relacionadas con turismo, consumo de restaurantes tradicionales y de lujo, en menor medida en cafeterías.

Se utilizará como técnica de captación de datos a la encuesta y se seleccionarán un número determinado de cafeterías representativas de la ciudad de Guayaquil. Se correrán las encuestas inicialmente por medio de un piloto que permita corroborar cada una de las preguntas planteadas y así minimizar errores que pudieran presentarse en la aplicación de la encuesta definitiva, con ello se observarán aquellos errores atípicos al correr los datos en el sistema estadístico (SPSS). Para establecer las relaciones de dependencia entre las variables 
de estudio, es necesario aplicar un análisis basado en el modelo de ecuaciones estructurales, ya que por medio de ecuaciones lineales se determinará la dependencia o independencia de las variables.

Para medir la consistencia o confiabilidad y validez del instrumento de medición utilizado en la investigación, se utilizará la medida de Alfa de Cronbach, por medio de la determinación del coeficiente en el paquete informático SPSS.

En estudios previos se ha seleccionado una cafetería representativa del lugar investigado, dentro de la presente investigación se determinará la factibilidad de realizarlo de manera similar o seleccionar de la población objetivo una muestra de cafeterías. En cuanto a la selección de los encuestados se aplicará en instrumento a un número determinado de clientes que concurran a las cafeterías y únicamente será aplicable a aquellos individuos que han realizado la compra en ese momento, de esta manera se garantiza que son consumidores de la cafetería. La selección de la muestra estará dada por un número de compradores de las cafeterías, los cuales serán seleccionados de manera aleatoria en diferentes horarios, siempre que tengan la predisposición para contestar la encuesta; en consecuencia, se utilizará un muestreo sistemático para lograr aleatoriedad en la recolección de datos.

Los criterios de inclusión para el desarrollo de la encuesta son: consumidores que efectúen compras, consumidores hombres y mujeres de cualquier edad, consumidores que asistan al establecimiento en cualquier momento del día. Los criterios de exclusión son: personas que ingresen al local, pero no efectúen la compra.

Las escalas que se utilizarán para las preguntas que se encuentren planteadas en el cuestionario serán de tipo Likert, para medir cada una de las preguntas.

Se medirá la confiabilidad y validez para finalizar con la comprobación del modelo por medio de ecuaciones estructurales. En tal sentido, se plantea dividir el estudio en constructos de orden que responderán a las relaciones existentes con la variable investigada y cada uno de ellos tendrán dimensiones; las mismos que han sido basados en la revisión bibliográfica de investigaciones previas que modelan el comportamiento del consumidor mediante la utilización de diversas variables. A continuación, se presenta de manera resumida las fases a aplicarse en el modelo de ecuaciones estructurales. 
Tabla 3. Fases del Modelo de Ecuaciones Estructurales.

\section{Fases para aplicar la técnica}

1. Especificación

2. Identificación

3. Estimación de Parámetros

4. Evaluación del Ajuste

5. Reespecificación del modelo

6. Interpretación de Resultados

\section{Descripción}

Relación entre variables latentes y observadas. Identificación de parámetros del modelo.

Determinar parámetros desconocidos.

La bondad de ajuste determinará si es correcto el modelo (ajuste global, incremental ajuste de parsimonia).

Permitirá conocer si el modelo obtenido inicialmente es el mejor o generar un modelo con ajustes al inicial.

Para concluir la investigación se determina el modelo correcto y por lo tanto la aceptación o negación de las hipótesis.

Fuente: basado en Escobedo et al. (2016)

Para determinar las cafeterías ubicadas en la ciudad de Guayaquil, se ha realizado levantamiento de información de las bases de datos que se encuentran publicadas en la Superintendencia de Compañías, Valores y Seguros, siendo un organismo que controla las actividades de las compañías; se seleccionarán las empresas que se encuentran dentro de la clasificación: "Actividades de preparación y servicio de bebidas para su consumo inmediato en: cafés, tiendas de jugos de fruta, vendedores ambulantes de bebidas, etcétera", en donde se obtendrá información de la empresa principalmente de campos tales como: Registro Único de Contribuyentes, objeto social, tipo de empresa, fecha de constitución, provincia, cantón, ciudad, dirección. Posteriormente se seleccionan las empresas, acorde a su objeto social relacionado con la investigación y a la ciudad donde se encuentra. Para determinar cuántas sucursales presenta cada cafetería es necesario ingresar a la base de datos disponible en el Servicio de Rentas Internas y se seleccionarán aquellas cafeterías con sucursales que se encuentren activas, así como también la ubicación geográfica de ellas.

En la metodología utilizada en estudios anteriores, se observa que se seleccionan una o dos cafeterías reconocidas de la ciudad y con ellos se aplica la encuesta piloto a las sucursales, para posteriormente efectuar una encuesta validada, cuyo número de encuestados oscila 
entre 350 a 500 encuestas aproximadamente, en donde se tuvieron que validar los cuestionarios para definir el número de encuestas válidas.

Las preguntas presentadas en la encuesta estarán basadas en investigaciones previas relacionadas y adaptadas al contexto estudiado, así como también es necesario incorporar preguntas que permitan medir la racionalidad del consumidor, para ello se elaboraron preguntas que serán revisadas previamente por expertos en el área económica, sicológica y estadística, ya que en las investigaciones previas no se han evidenciado la incorporación de variables relacionadas.

\section{CONCLUSIONES}

En la revisión bibliográfica se ha obtenido la fundamentación teórica, metodologías utilizadas en investigaciones previas que se relacionan con la temática estudiada. En función de un levantamiento preliminar de información relevante se ha estructurado una propuesta metodológica que permita levantar información por medio del establecimiento de variables que se seleccionarán en el estudio para aplicarse en un contexto local (ciudad de Guayaquil) los patrones de comportamiento en el consumo, específicamente en las cafeterías y aquellas motivaciones de compra. Con el estudio se pretende establecer una base de análisis que permita determinar las variables que influyen en la compra de otros negocios.

Con frecuencia el análisis del comportamiento del consumidor es analizado bajo ciertos criterios de racionalidad con la aplicación de diversos supuestos que en muchas ocasiones permiten facilitar el análisis; sin embargo la existencia de una economía dinámica en donde la evolución del individuo expone a empresarios e investigadores a cometer ciertas omisiones en dichos análisis por medio de la aplicación de las teorías existentes, por ello se propone realizar un estudio que permita reconocer aquellas variables que influyen y que generan una fuente de información valiosa para los negocios con la aplicación de estrategias enfocadas al comportamiento actual del consumidor, permitiendo así incrementar su volumen de ventas y en contrapartida logrando incrementar los niveles de satisfacción del cliente porque se logrará comprender los incentivos y necesidades reales.

Existen modelos formales de economía que presentan características generales o patrones de comportamiento en el caso de la conducta de los consumidores y como consecuencia de aquello nacen propiedades, los cuales han sido válidos en ciertos contextos, pero que no han 
sido reevaluados con sus cambios o modificaciones de la evolución del ser humano y su cambio en tendencias de consumo, costumbres, gustos y preferencias, es decir que no han sido adecuadas por la influencia de histórica, tomando en consideración el tiempo como factor de valoración para la observación de variaciones relevantes.

En la revisión de estudios previos la metodología utilizada ha sido por medio la aplicación de encuestas a los clientes de los negocios a analizarse fueron: cafeterías, hoteles tradicionales y temáticos: en donde principalmente los estudios coincidían en el análisis de variables tales como: percepciones, lealtad, personalidad, ambiente del negocio, cuyo enfoque fue cuantitativo, correlacional y transversal con muestreo aleatorio y sistemático, en donde se establece un modelo por medio de ecuaciones estructurales que permitan establecer si se aceptan o rechazan las hipótesis planteadas.

El presente documento ha planteado la fundamentación teórica e investigaciones previas relacionadas con el enfoque de estudio, en donde principalmente se ha planteado la metodología a utilizarse y aquellas variables preliminares de estudios en el modelo que se utilizará por medio de ecuaciones estructurales. En el transcurso de la investigación se espera abordar a profundidad el estudio y la propuesta del modelo en donde se generé una mayor contribución a estudios relacionados.

\section{REFERENCIAS BIBLIOGRÁFICAS}

Borrás, I. C. V. (2004). Psicología económica y del comportamiento del consumidor. Retrieved from https://ebookcentral.proquest.com

Berenguer, C. G., \& Gómez, B. M. Á. (2006). Comportamiento del consumidor. Retrieved from https://ebookcentral.proquest.com

Chen, A. \& Peng, N. (2018). Examining consumers' intentions to dine at luxury restaurants while traveling. International Journal of Hospitality Management (p. 59-67).

Devesa, M., Laguna, M. y Palacios, A. (2010). Motivación, satisfacción y lealtad en el turismo: el caso de un destino de interior. Revista Electronica de Motivacion y Emocion Monográfico de la Motivación del consumidor: Estudios experimentales, XIII (35-36), 22. Obtenido de http://reme.uji.es/articulos/numero35/article9/article9.pdf 
Escobedo, M. T., Hernández, J. A., Estebané, V. y Martínez, G. (2016). Modelos de ecuaciones estructurales: Características, fases, construcción, aplicación y resultados. $\begin{array}{llll}\text { Ciencia } & \& & \text { trabajo, } & 18(55),\end{array}$ https://dx.doi.org/10.4067/S071824492016000100004

Fernández, E. (2012). La motivación en la toma de decisiones: una concepción alternativa. revista de ciencias sociales. 18(1), 2012. Retrieved from https://ebookcentral.proquest.com

Forgas, S., Moliner, M., Sanchez, J. y Palau, R. (2011). La formación de la lealtad de un cliente de una compañia aerea: diferencias entre aerolíneas tradicionales y de bajo coste. Elsevier España, S.L, 14(162-172), 11. Obtenido de https://ac.elscdn.com/S1138575811000119/1-s2.0-S1138575811000119main.pdf?_tid=d7cea295b211-4208-829e-

89bcdf62a63c\&acdnat=1534725450_a56c81e821800a342783f169b70eeab8

Heesup Han \& Kisang Ryu (2009) The Roles of the Physical Environment, Price Perception, and Customer Satisfaction in Determining Customer Loyalty in the Restaurant Industry. Journal of Hospitality \& Tourism Research, Vol. 33, issue: 4, (p. 487-510)

Jang-Hyeon Nama, Timothy Jeonglyeol Lee (2011). Foreign travelers' satisfaction with traditional Korean restaurants. International Journal of Hospitality Management (p. 982-989)

Jeannot, R. F. (2006). Hacia el estudio empírico de los comportamientos. Retrieved from https://ebookcentral.proquest.com

Lee (2018). The Influence of the Experiential Marketing Factors of Restaurant on the Brand Image, Satisfaction, and Customer Loyalty: Focused on Restaurants in Complex Shopping Mall. Culinary Science \& Hospitality Research, 24 (2), 112- 118.

Matos, R. F. y Martín, S. S. (octubre-diciembre de 2012). Análisis sobre la reputación de marca,las emociones y la confianza como formadoras de la satisfacción del turista. Redalyc.Contaduría y Administración, 57(4), 35. Obtenido de http://www.redalyc.org/pdf/395/39524375012.pdf

Moliner, B. y Berenguer, G. (enero-junio de 2011). EL EFECTO DE LA SATISFACCION DEL CLIENTE EN LA LEALTAD: APLICACION EN ESTABLECIMIENTOS MINORISTAS. 
Scielo (101-124), 24 Obtenido de

http://www.scielo.org.co/pdf/cadm/v24n42/v24n42a05.pdf

Moliner, B., Berenguer, G. y Gil, L. (2001). LA IMPORTANCIA DE LA PERFORMANCE Y LAS EXPECTATIVAS EN LA FORMACIÓN DE LA SATISFACCIÓN DEL CONSUMIDOR. Investigaciones Europeas de Dirección y Economía de la Empresa, 7(3), 18. Obtenido de

/Dialnet-LalmportanciaDeLaPerformanceYLasExpectativasEnLaFo206181.pdf

Petr Štumpf, Viktor Vojtko, Barbora Valtrová (2018). Satisfaction of European Tourists - Benchmarking of EU Countries. Global Business \& Finance Review (p. 114).

Roger, H. (1996). The relationships of customer satisfaction, customer loyalty, and profitability: an empirical study. International Journal of Service Industry Management, 7(4), 27-42. doi: doi:10.1108/09564239610129931

Ruiz, M. y Palaci, F.J. (noviembre de 2011). VARIABLES COGNITIVAS Y PSICOLOGÍA DEL CONSUMIDOR: El modelo de la confirmación de expectativas en la actualidad. Boletin de Psicologia (103), 13. Obtenido de https://www.uv.es/seoane/boletin/previos/N1034.pdf

Sergueyevna, N., \& Mosher, E. L. (2013). Teorias motivacionales desde la perspectiva del comportamiento del consumidor. Revista cientifica electronica de ciencias gerenciales (26), 14. Obtenido de /226-599-1-SM.pdf 